\title{
Théologiques
}

Théologiques

\section{Par ici la sortie}

\section{Ce qu'il advient de la spiritualité dans l'horizon du langage}

\section{Étienne Pouliot}

Volume 18, numéro 2, 2010

Les lieux de la spiritualité aujourd'hui

URI : https://id.erudit.org/iderudit/1007483ar

DOI : https://doi.org/10.7202/1007483ar

Aller au sommaire du numéro

\section{Éditeur(s)}

Faculté de théologie et de sciences des religions, Université de Montréal

ISSN

1188-7109 (imprimé)

1492-1413 (numérique)

Découvrir la revue

Citer cet article

Pouliot, É. (2010). Par ici la sortie : ce qu'il advient de la spiritualité dans l'horizon du langage. Théologiques, 18(2), 121-142.

https://doi.org/10.7202/1007483ar
Résumé de l'article

L'adoption d'une perspective herméneutique plutôt que phénoménologique permet de décrire le " spirituel » en évitant toute substantialisation à cet égard, tant de « la spiritualité » elle-même que de ses soi-disant composantes. Une dépsychologisation devient ainsi possible, au bénéfice d'une présentation en termes de pratique de spiritualité (par opposition à pratiques spirituelles), de geste de la parole (en son titre même de spiritualité) et de pratique signifiante fondamentale.
Ce document est protégé par la loi sur le droit d'auteur. L'utilisation des services d'Érudit (y compris la reproduction) est assujettie à sa politique d'utilisation que vous pouvez consulter en ligne.

https://apropos.erudit.org/fr/usagers/politique-dutilisation/ 


\title{
Par ici la sortie
}

\section{Ce qu'il advient de la spiritualité dans l'horizon du langage}

\author{
Étienne Pouliot" \\ Théologie et sciences religieuses \\ Université Laval
}

L'extase dont nous entretiennent tant de mystiques demeure un phénomène ou même une vérité dont on peut discuter. La question est de savoir comment le faire, en quels termes, selon quel cadre, en vue de quoi... Maurice Zundel parlait de la mystique en général et de cette fameuse extase en particulier comme d'une diction de soi en la connaissance de soimême, dans et par un Autre (Zundel, 1962). Déjà, me semble-t-il, une pareille formulation suggère un déplacement dans la manière de traiter et donc de comprendre cette "sortie de soi »; encore faut-il lâcher prise quant à ce qui peut nous retenir de saisir le mouvement même annoncé là, en cette sortie, par un mystique chrétien ou par un homme tel que Maurice Zundel.

C'est d'une façon plus ordinaire toutefois que je rendrai compte de cet enjeu de sortie ou de passage - pourquoi pas d'accès ou d'entrée, selon qu'on voudra l'envisager. Quittant l'horizon mystique, qui connote éminemment l'exceptionnel ou l'extraordinaire, je m'en tiendrai au champ de la spiritualité, ce dont tous et chacun peut se réclamer. Je compte le faire non pas à partir de la littérature sur la spiritualité mais sur la base d'une expérimentation, d'une situation concrète: celle d'un colloque réalisé sur la question de la spiritualité dite laïque. Cette question n'est d'ailleurs pas étrangère au problème que je souhaite éclairer; je m'en expliquerai en temps et lieu.

* Étienne Pouliot est chargé d'enseignement à la Faculté de théologie et de sciences religieuses de l'Université Laval. Ses champs de recherches portent sur l'éthique et la spiritualité, l'approche sémiotique des textes (religieux ou non) et le dialogue philosophie et théologie. Il a récemment publié É. Pouliot et A. Fortin (2009), Re-cueillir la Parole. Une lecture sémiotique de récits évangéliques, Montréal, Novalis. 
Je veux montrer que nos tentatives actuelles pour cerner la spiritualité, et par conséquent les conceptions habituelles qu'on s'en donne, sont aujourd'hui devenues plus secondaires et réductrices qu'il n'y paraît. De ce point de vue, il s'agit bel et bien d'indiquer une sortie de certaines perspectives pour entrer dans une autre, soit celle d'une philosophie du langage. Je cherche à décrire la spiritualité de manière liminaire pour en offrir une vue simple (mais non simpliste) et contrastée (par rapport à nos évidences actuelles); j'ai le souci d'en présenter une vue autrement fondatrice en raison du seuil (frontière, limite entre deux espaces, à la fois sortie et entrée) qu'elle constitue pour une existence proprement humaine (et herméneutiquement réalisée) ${ }^{1}$. En ce sens, il s'agit de saisir à sa racine ce qu'on appelle «la » spiritualité ou, ce qui revient au même, d'exhiber le geste qui dit et fait tout à la fois une spiritualité en chacun et pour chacun. Car la manière originaire de penser et de vivre une spiritualité a trait à une pratique: qui "fait la spiritualité». Ainsi, notre propre inscription dans la chair, dans un réel qui n'échappe pas à de l'imaginaire et du symbolique ${ }^{2}$, nous faire advenir en spiritualité; est concrètement spirituel ce qui advient

1. Ma proposition procède d'un arrière-plan théorique et philosophique certain, dont voici les grandes lignes. Mon propos se situe dans l'horizon langagier de la philosophie moderne et plus spécialement dans le sillon d'une sémiotique exposant les conditions de l'énonciation, avec sa théorie propre de la signification (Delorme 1992; Panier 2008). Il vise à dégager la spiritualité de l'approche ontologique-métaphysique puis phénoménologique qui lui a été globalement accolée - non sans fruits, il faut en convenir. Plus précisément, je prends mes distances avec l'idéalisme qui marque la phénoménologie husserlienne, dont la pensée de Paul Ricœur garde les traces évidentes, et me rapproche de l'herméneutique heideggérienne et plus encore gadamérienne dans l'exacte mesure où s'y exhibe un enjeu interprétatif (herméneutique plutôt que phénoménologique) allié à la médiation incontournable et sans pareille du langage. Ceci m'éloigne, par le fait même, de l'herméneutique "psychologisante » à la manière de Schleiermacher ou de Dilthey; c'est tout de même de Feuerbach dont je me démarque par-dessus tout, avec son projet qui clôture et clôt l'herméneutique classique - avis que je partage avec Jean-Pierre Osier dans sa préface à l'ouvrage-clé de Feuerbach (1982 [1841]). Ce sont donc les notions d' "expérience ", de "vécu ", de «données » (de l'expérience), de la "tradition », du "sujet» et d' "objet », outre le statut du savoir, qui sont au cœur de la sortie d'une approche somme toute toujours prédéterminée - voire déterministe - de la spiritualité. Et mon propos consistera à signaler vers quoi se tourner quand ces notions changent d'importance ou même de sens.

2. La formule renvoie à la perspective que Jacques Lacan (1975) a proposée de cette triple structure langagière du Réel, de l'Imaginaire et du Symbolique. Cette structure signifie que rien n'est jamais un «en-soi », en vertu d'un certain savoir sur les choses; tout est inextricablement lié, tels des anneaux borroméens. 
dans et par la parole, en acte de parole, comme parole incarnée: en la trace d'un Autre ainsi manifesté... peut-être même pas énoncé. Reprenons tout ceci.

\section{Mise en situation: un colloque portant sur la spiritualité dite laïque}

Je commencerai par illustrer le sens global de mon propos en recourant à l'événement d'un récent colloque qui, à mon avis, a constitué une expérience, celle d'un véritable dialogue, sur une question qui éclaire justement le problème (et l'issue) que j'ai en vue. J'en indique d'abord les orientations puis en dégage des considérations liminaires pour situer et concevoir autrement «la spiritualité».

En avril 2009, un colloque fut organisé par la revue des Cahiers de spiritualité ignatienne produite par le Centre de spiritualité Manrèse de Québec, en collaboration avec la Faculté de théologie et de sciences religieuses de l'Université Laval. Depuis deux ans déjà, un questionnement sur la «spiritualité laïque » avait surgi de la démarche de perfectionnement des personnes collaborant au Centre. Ce questionnement prenait toute son importance pour deux raisons. D'abord, le Centre propose, depuis sa fondation en 1976, une démarche originale pour vivre les Exercices spirituels de saint Ignace de Loyola; la mission spécifique du Centre consiste à offrir ces Exercices dans la vie courante (EVC), pour les personnes laïques nommément. Ensuite, de plus en plus de laïques sont impliqués, à titre de personne accompagnatrice, dans cette offre au public.

Au cours de l'année précédente, deux numéros des Cahiers avaient été consacrés à cette question de la spiritualité dite laïque, en préparation directe au colloque. Un premier numéro, de nature exploratoire, permettait à des personnes plus ou moins liées à l'Église de parler de la façon, nouvelle ou mieux adaptée à leurs besoins (selon elles), dont elles cherchent à vivre leur vie spirituelle à travers leur engagement social et communautaire (Cahiers 2008). Un second numéro était consacré au discours spirituel non religieux; des personnes se considérant sans lien avec une tradition religieuse déterminée, c'est-à-dire des gens qui se disent athées, incroyants ou simplement humanistes parlent de la spiritualité dont ils se réclament encore (Cabiers 2009a).

Par conséquent, le colloque sur la spiritualité dite laïque visait, d'une part, à aborder la question d'une façon différente du laïcisme anti-religieux ou du moins se déclarant areligieux. Il s'agissait, d'autre part, de prêter attention à des manières originales de vivre la spiritualité chrétienne actuel- 
lement, sans répéter la problématique d'un autre colloque des Cahiers, tenu en 2004, sur le thème apparenté de l'arrimage entre laïques et personnes consacrées; là, la question des charismes spécifiques d'une tradition ou d'une communauté religieuse en vue d'examiner leur reprise éventuelle, notamment par des "associés", avait été traitée (Cahiers 2005). Le colloque 2009 des Cabiers portait donc sur une problématique proche de ce questionnement relatif à l'héritage direct et explicite des religieux et religieuses au Québec; la place et le rôle des religieuses et des religieux devaient être resitués expressément en fonction de la présence dans le monde, conformément à ce qui définit couramment le laïcat depuis le $\mathrm{xx}^{\mathrm{e}}$ siècle.

Les principales questions à discuter lors de ce colloque étaient les suivantes. Quelle inscription autre dans la tradition spirituelle chrétienne est rendue nécessaire de nos jours, attendu que la vie monastique et même l'expérience mystique ${ }^{3}$ ne constituent plus le modèle de la spiritualité — ni sa référence explicite ni sa norme première? En quoi consiste au juste "notre spiritualité », comme personnes laïques en Église, une spiritualité encore souvent et habituellement qualifiée de «séculière » mais qu'il semble falloir nommer autrement pour diverses raisons? Et au compte de ces raisons, un avènement et une condition majeure: quel est donc ce lieu de la spiritualité qu'est devenu le monde contemporain? Pour ce colloque, la problématisation de la «spiritualité laïque» fut donc centrée sur la prise de parole des personnes laïques elles-mêmes et axée sur leur propre situation, à savoir habiter le monde d'aujourd'hui.

Il ne sera pas inutile de donner ici un aperçu du contenu des quelques conférences prévues au programme, les actes complets de ce colloque ayant d'ailleurs été publiés (Cahiers 2009b). Deux conférences ont ouvert la réflexion. La première concernait la situation et le destin de l'Action catholique, en écho au Rapport Dumont (1971); elle signalait les rendez-vous manqués et faisait émerger des pistes d'espérance quant à la mise en place, par les laïcs engagés dans le monde, d'une spiritualité autrement vécue en Église. L'autre conférence traitait des enjeux quant à une transformation de la spiritualité chrétienne au XviI ${ }^{\mathrm{e}}$ siècle; le discours et l'expérience des spirituels se proposaient dorénavant comme subjectivité auto-affirmée, pour passer plus nettement par l'expérience, l'intériorité, le corps. De nombreuses méprises sur cette époque furent alors élucidées, notamment en ce

3. «Mystique» au sens où on l'entend couramment aujourd'hui et depuis le XIX siècle, en parlant des sommets exceptionnels de la vie chrétienne (Agaesse et Sales, 1932). 
qui concerne ces mêmes catégories aujourd'hui devenues réductrices en raison de la mentalité scientifique actuelle. Par la suite, deux recherches "appliquées» ont fourni à ces perspectives une base exploratoire davantage accessible. On a ainsi pu réfléchir à la vie spirituelle au quotidien, dans une quête de petits moments spéciaux et de rites réinventés pour marquer la vie ordinaire et redéployer des «liturgies" séculières. On a pu, en outre, s'enquérir de la démarche des Exercices spirituels dans la vie courante, pour la dégager de l'idéal classique de la retraite intensive de trente jours et donc déployer le tout en une démocratisation de la dynamique de groupe à travers une éthique de la parole.

En outre, des ateliers d'échange en petits groupes ainsi qu'une table ronde avec des personnes chargées de dresser une sorte de bilan prospectif du colloque ont assuré, d'une autre façon, une circulation de la parole pendant tout le colloque.

J'examine à présent en quoi cette situation (un colloque) et ce thème (la spiritualité dite laïque) illustrent une mise en œuvre de la spiritualité qui est aussi significative en elle-même - voire plus significative encore - que ce qu'on a pu alors dire sur la spiritualité. Mon analyse s'orientera sur le phénomène de (prise de) la parole et mettra en relief des enjeux proprement herméneutiques signalant des renversements de perspective.

\section{Objet-et-sujet de spiritualité, en un geste de parole}

Pour qui a participé à ce colloque ou qui prend connaissance en détail des Actes de colloque, une chose au moins est sûre: on a refusé de définir la spiritualité à partir de contenus, dits spirituels. Les "choses spirituelles", les affaires de Dieu ou de l'Esprit, les repères chrétiens habituels de toute expérience dite spirituelle, les thèmes mêmes de la vie spirituelle, rien de cela n'a servi à fournir une base commune ou un point de départ ferme pour les échanges. Aucun accord initial n'a été recherché ni établi autour d'une sorte d'universel («La spiritualité») dégagé à partir de conceptions particulières et donc des expériences personnelles propres de la spiritualité.

Pareille approche, pareille disposition a de quoi indisposer. En effet, les contenus et thèmes abordés pendant ce colloque, en particulier lors des ateliers, ont à ce point varié qu'on peut se demander à quoi rime le colloque dans son ensemble. On y passe des enjeux sociaux aux expressions liturgiques, de la prière et de l'expérience personnelle aux convictions religieuses et politiques (au sens large du terme) sur le monde moderne et sur la vie humaine. À l'évidence, il ne s'agissait pas de prendre position sur 
ceci ou cela et en fin de compte de définir la spiritualité — sinon une spiritualité, sa spiritualité.

La véritable rencontre, si l'on veut bien acquiescer à cette prétention quand on n'a pas vécu l'événement, s'est produite autrement... ou ailleurs. Elle est advenue et s'est maintenue dans et par la prise de parole des participants. Au lieu d'en rester à des notions en fonction desquels établir un accord du même ordre, les personnes participantes ont pris une place et mis en œuvre leur spiritualité, qui se signale aussi avec des savoirs à monnayer entre elles. Une prise de parole permettait de rendre disponibles des contenus, d'ordre clairement spirituel ou autre; des conceptions de la spiritualité et des expériences à cet égard ont pu s'exprimer, mais c'est la prise de parole - possible et effective pour chacun — qui demeure le geste originaire d'où tout se met en branle. Ce serait trop réducteur de considérer le colloque comme une scène pour l'avènement des sujets, bien qu'il y ait du vrai à penser de la sorte; ce serait trop peu dire d'y voir seulement quelque effet de subjectivation puisqu'il en va d'un geste qui fait beaucoup plus: en disant l'objectivité tout aussi concrète de l' 'à-faire» spirituel. Manifestement, la parole instaure un lieu commun — avec des références, des connaissances, des attitudes - qu'elle constitue fondamentalement comme un geste pour le moins complexe: celui de prendre place, d'avoir son moment, de se mettre en œuvre, de rassembler et d'énoncer. La difficulté, si elle demeure, c'est de nommer déjà «spiritualité » la parole désignée - oui, désignée! - comme geste et geste originaire.

Un renversement magistral, de perspective et de méthode (au sens où odos, dans methodos, signifie chemin et accès), préside à une telle analyse... ou peut seul la valider. Il est possible de parler de spiritualité avant toute désignation de la «chose spirituelle» — sans en principe exclure ceci ni en faire fi en pratique, comme je l'expliquerai plus loin. La détermination spécifique des choses, suivant un mode de définition de l'essence de chaque chose dans une perspective (onto-)métaphysique classique, n'est pas la garantie de la vérité au plan spirituel — ou autre, ajouterais-je — et pas plus de l'authenticité d'une spiritualité; le propos des mystiques modernes permet d'en convenir clairement ${ }^{4}$. Par ailleurs, le fait de parler de «choses spirituelles» ne garantit pas non plus une spiritualité ${ }^{5}$. La

4. Voir par exemple la défense, par Jean-Joseph Surin (1963 [1661]), de la mystique (moderne) alors qu'elle émerge.

5. Ludwig Feuerbach (1982 [1841]) en est un des exemples contemporains les plus notoires. Réagissant à son maître Hegel et à sa phénoménologie de l'Esprit absolu, Feuerbach les retourne et expose la pure rhétorique de la vérité qui constitue le res- 
racine de la spiritualité se trouve ailleurs: en un geste, le geste de la parole — manifestée bien sûr avec le discours, ce que je considérerai plus loin.

Ce renversement signale un autre statut accordé aux objets dans le régime de la connaissance humaine, dont spirituelle. Loin d'être évacuée, l'objectivité en demeure la condition sine qua non. Mais cela ne saurait renvoyer ni équivaloir à des objets figés dans un ordre fixé d'essences, à des choses-en-soi. La mystique aussi bien que la philosophie modernes représentent un seul et même pas à cet égard, faisant entrer l' "expérience » dans le régime même de la connaissance pour la fonder avec ses objets phénoménaux; on ne peut plus revenir en arrière sans rater, sans se méprendre sur le sens de la modernitée ${ }^{6}$ À son tour la phénoménalité, c'està-dire les objets phénoménaux, se révèle en son caractère herméneutique au fur et à mesure que l' "expérience » spirituelle (mystique mais aussi ordinaire) s'affiche et est finalement davantage comprise dans sa consistance langagière ${ }^{7}$. Dès lors qu'objectivité et médiation — qu'on en parle en

sort de l'interprétation jusqu'à son époque. Interpréter, c'est alors dire en d'autres mots le sens vrai, déjà là tout donné, dans le religieux. En vertu de la technique interprétative feuerbachienne (qui n'a encore rien de proprement herméneutique, au sens d'une philosophie herméneutique moderne qui sera d'ailleurs une des issues à un Hegel prétendant avoir définitivement parachevé la philosophie), le christianisme nommément se réduirait à ou en des jeux de mots à l'intérieur d'un mécanisme interprétatif fort simple qui exclut toute spiritualité par-delà l'humain et toute vérité autre que ce que livre un énoncé. On aurait tort de se croire exempt de la critique feuerbachienne; un discours religieux athée, sous couvert de notions spirituelles apparemment évidentes et donc "qui crèvent les yeux ", peut très bien être notre lot - comme le rappelle encore Jean-Pierre Osier, toujours dans la préface de l'ouvrage maître de Feuerbach (1982 [1841]).

6. C'était nettement le projet de la Critique de la raison pure de Kant (1976 [1781]), outre celui des mystiques modernes dont Surin, déjà évoqué. Il est intéressant de mentionner que le "réalisme" des sciences modernes a été poussé à un point où le matériel et le spirituel se joignent; ainsi l'objectivité pure et absolue et le positivisme le plus ferme s'évanouissent avec la théorie des quanta (Heisenberg 1972 [1932]; on peut en trouver une présentation simplifiée dans Zundel 1948 [1944]).

7. On pourrait comparer le propos de différents mystiques (Maître Eckhart, Jean de la Croix, Surin) pour vérifier dans quelle exacte mesure le statut du langage s'y trouve exposé, en une théorie en bonne et due forme. On doit clairement à Michel de Certeau d'avoir souligné cela, en montrant ou en rappelant à quel point la notion de langage, plus que celle d'expérience ou parce que celle d'expérience l'inclut déjà toujours, permet de rendre compte de la mystique moderne et, plus largement, de la spiritualité (par exemple: De Certeau, 1982 et 2005). Dans l'univers de la philosophie moderne, tous reconnaissent au moins l'inflexion herméneutique que Ricœur a voulu donner à la phénoménologie, mais on ne peut s'en suffire - et c'est tout le sens de mon propos. 
termes d'historicité, de conditions de validité, de langage — s'allient inextricablement et cessent d'être dichotomisées ou même opposées, un "réalisme de structure» devient pensable et assure de prendre acte de ceci: tout est interprétation ${ }^{8}$. À titre de spiritualité, le geste de la parole fait donc porter tout l'enjeu de l'objectivité sur notre rapport, sur le rapport même au réel: en faisant de nos objets ce que nous en construisons en termes de contenus, d'idées, de références. Et à ce compte, Dieu apparaît enfin et il demeure où devait demeurer l'objet par excellence: l'objet le plus à construire par nous pour exister en nous sans se réduire à nous. Les mystiques nous ont-ils indiqué autre chose que ce rapport, que ce mouvement vers «l'Objet » un tant soit peu compris dès lors qu'il s'élabore en nous et par nous ${ }^{9}$ ?

Le sens (direction) de ce renversement attesté par la démarche du colloque en question l'est tout autant chez les maîtres spirituels. Il en va de l'articulation du sujet à son objet, d'un sujet advenant en spiritualité avec son objet, comme lui et en même temps que lui, pour être autre ${ }^{10}$. Ni sujet ni objet d'emblée constitués, mais un procès radical de l'un comme de l'autre. Subjectivité et objectivité comme co-exigences radicales. La réalisation ou plus exactement la structuration d'une identité de part et d'autre, par opposition à une identité définitivement établie selon des termes toujours déjà substantiellement définis. Une compréhension décentrée à la fois du monde et de soi, par opposition à une conception de soi et du monde idéalisés en vertu de quelque essence déterminée. Tout, en spiritualité: manifesté comme tel et concrètement tel. Bref, un geste qu'on pourrait dire de foi et réel, tenant à/par cette contemporanéité toute spirituelle et tout à fait concrète du sujet et de son objet. Un geste, qui est bien celui de la parole. Car un acte de parole ne fait pas simplement attribuer un prédicat à un sujet logique ou déployer des contenus de savoir; en son titre de spiritualité concrète, il fait l'objet pour un sujet et vice versa. Car la parole (le

8. J'emprunte l'expression « réalisme de structure» à Maurice Zundel (1948 [1944]), mais c'est du post-structuralisme dont il en va. De même, l'idée selon laquelle tout serait interprétation provient de Feuerbach (1982 [1841]), mais c'est tout le poids de l'herméneutique moderne que j'évoque.

9. D’où les thèmes de la lumière prenant le pas sur les ténèbres sans jamais les évacuer, propre à la mystique moderne - par opposition à la tradition spirituelle antérieure où c'est plutôt l'inverse. Michel de Certeau (2005) montre l'impact de prendre Augustin ou bien Denys l'Aéropage dans la manière de concevoir la spiritualité avant et à partir des $\mathrm{XVI}^{\mathrm{e}}-\mathrm{XVII}^{\mathrm{e}}$ siècles.

10. Maurice Zundel a parlé de «co-naissance», en jouant donc avec l'étymologie du mot. 
langage) n'est pas un véhicule de la pensée sur des choses - enjeu purement théorique, avec son tribut à l'idéalisme -, mais un acte où sujet comme objet se trouvent constitués et donc ensemble engagés; elle n'est pas un simple moyen d'expression - enjeu esthétique, avec sa rançon du subjectivisme -, mais un avènement de sujets d'objets. Dans ce surgissement d'un sujet en relation à son objet, l'épineuse question de la projection de soi au cœur d'une expérience humaine, dans le champ religieux entre autres, n'est pas ignorée ni surtout niée mais herméneutiquement assumée et traversée ${ }^{11}$.

On en reste donc, avec le geste de la parole, à quoi il s'agit précisément d'accorder le titre de spiritualité, on en reste à un véritable procès de sujets et d'objets, pour faire la vérité sur les uns et les autres en même temps. Reste à examiner comment certaines notions maîtresses de "la spiritualité » sont pareillement affectées quand on la comprend et l'enracine dans l'horizon du langage.

\section{Au travers du vécu, de l'expérience et de l'intériorité}

Les échanges au cours du colloque qui sert de point de départ aux présentes réflexions n'ont pas été occupés par du «vécu » à exprimer et à partager. Par le fait même, on n'a guère été préoccupé de présenter, encore moins de rendre compte de "sa spiritualité " (à chaque individu ou groupe) et encore moins de faire valoir une spiritualité spécifiquement laïque. Il n'est pas du tout insignifiant de le relever si tant est que la question du "vécu» apparaît de nos jours comme habituelle, centrale, déterminante pour juger une "expérience spirituelle». De façon générale, pour nos contemporains, le vécu a pris la valeur d'évidences, de certitudes ou de convictions intérieures, qui manifestent ou attestent une expérience spirituelle valorisée dans ses spécificités, sa particularité ou encore son universalité.

Or, cette notion de "vécu » est sans aucun doute tributaire d'une conception incontestablement déterminée, et qui plus est déterministe, de l'expérience, qui doit alors comporter des «données» repérables et par suite analysables. Tout repose ainsi sur de prétendus constituants de la conscience, sur des éléments constitutifs particuliers ou universels de celle-

11. Je renvoie aux projets critiques de Feuerbach et, bien sûr, de Freud, qui mettent fin au déni de l'illusion, mais c'est à celui de Jacques Lacan que je m'associe finalement parce qu'il dépsychologise l'analyse (psychanalyse) et rend cette démarche à la vérité du désir. Lacan déplace d'ailleurs tout le problème de l'être et de la communication (technicité du parler) en direction d'un «parlêtre». 
ci. Toute l'analyse de l'expérience spirituelle procède en vertu d'un idéalisme ou sinon d'un positivisme dans la sphère des sentiments humains ou, plus largement, de la conscience ${ }^{12}$. La notion même de vécus demeure peut-être un reliquat des essences/substances qui ont fait la gloire de la métaphysique classique; chose certaine, les "vécus" représentent une claire psychologisation - en vertu de déterminismes, faut-il le répéter de l'expérience spirituelle, particulière ou tenue pour universelle. La spiritualité est ainsi axée sur des affects et il ne reste souvent plus qu'à sauvegarder la qualité proprement spirituelle ou chrétienne de l'expérience, par quelque référence spéciale à Jésus, à Dieu, à l'Esprit, aux choses considérées comme vraiment spirituelles (c'est-à-dire prédéterminées telles).

Ceci explique en grande partie la redécouverte, des dernières décennies, de la spiritualité dans l'univers des soins hospitaliers, par exemple. On en fait alors une dimension, parmi d'autres, de l'être humain et on l'analyse à partir de repères, normes et procédures issus des disciplines variées; la spiritualité devient cette dimension dite nécessaire, la plus haute ou la plus sublime qui soit, la plus profonde ou encore celle-là même qui doit être transversale aux autres dimensions biologiques, psychologiques, morales, sociales, intellectuelles (etc.) de l'humain. Aussi la vie intérieure comporterait-elle des données et des étapes semblables - empruntées ou dupliquées quant - à la vie biologique, psychologique, sociale... (Pouliot 2001). Loin de nier l'apport appréciable de cette approche pluridisciplinaire de la spiritualité, mon propos cherche par-dessus tout à mettre en relief le caractère second de celles-ci, par un renversement de perspective exposant le sens originaire et donc le caractère radicalement herméneutique de la spiritualité en sa mise en œuvre de parole.

Il faut se rappeler, par surcroît, que si le concept d'expérience peut inclure celui du vécu, il ne s'y réduit certainement pas $^{13}$. La démarche du

12. On doit à F. D. E. Schleiermacher et plus encore à W. Dilthey le grand projet d'une description de la sorte de l'expérience vécue (Erlebnis). Au tournant du XIX ${ }^{\mathrm{e}}$ siècle, les débats portaient d'ailleurs sur l'établissement des sciences de l'esprit, par opposition aux sciences dites dures (ou pures). Dilthey, en particulier, a voulu développer ces sciences de l'esprit en reprenant, en assumant les vues, démarches et outils des sciences pures. Hegel aura au moins émancipé les sciences de l'esprit des sciences pures, ligotant celles-là à un idéalisme/rationalisme totalitaire. La réduction phénoménologique husserlienne, ensuite, garde encore les traits d'un idéalisme déniant tout ressort herméneutique (au sens moderne du terme, pas au sens classique ou même feuerbachien du terme).

13. La langue française n'a qu'un mot pour exprimer ce que l'allemand distingue plus nettement: «expérience» au sens d'expérimentation et donc d'essais et de tâtonne- 
colloque a justement permis aux participants de se poser à distance de leur "expérience ", dont leur "vécu ", non seulement par une attitude critique (normale et requise dans les circonstances) mais en raison même de la prise de parole qu'elle a rendu possible et maintenue. Ce n'est donc pas un concept déterminé d'expérience qui a été retenu comme critère et point de repère pour ce colloque, ce dont les Actes attestent clairement par la diversité des thèmes abordés; c'est plutôt le fait d'une posture, le rapport luimême à son "expérience » ou à son "vécu ", la capacité de fonctionner de façon à manifester et instaurer des sujets naissant avec d'autres et dans la contemporanéité de leurs objets. La parole a institué certes des objets (points ou contenus de discussion), mais d'abord et surtout des sujets de spiritualité... avant des objets mais non sans eux. Et cet «avant» a un sens herméneutique plutôt que chronologique ou logique; cet "avent", cet avènement en parole appartient bien à une phénoménalité où le donné, objectif autant que subjectif, relève d'une structure d'intériorité propre à la parole et qui ne coïncide pas avec quelque déterminisme.

À cette notion d'intériorité, aussi, il faut faire subir un déplacement afin de l'émanciper du psychologisme qui le clôt «idéalistement » et trop vite de nos jours. Bien sûr, l'établissement de ce fait qu'est l'intériorité spirituelle demeure l'acquis le plus important et le plus notoire à l'aube de la modernité, alors que des maîtres spirituels exposent leur vie intérieure et cherchent à en rendre compte en elle-même, pour elle-même et par ellemême ${ }^{14}$. Il n'en demeure pas moins que cette donnée reste liée à l'émergence du paradigme de la conscience à l'époque moderne, à quoi répondait une conception psychologique de l'intériorité. L'expérience vécue (Erlebnis), la mise de l'avant de la subjectivité moderne, l'enquête phénoménologique relativement à la conscience et au réel, tout ceci n'a fait qu'accentuer l'intériorité dans son caractère déterminable, psychologique-

ments (Experiment, experimentum en latin), au sens de risque et péril dans une transformation/altération qui produit le sage-expert (Erfahrung, ce qui ne correspond pas tout à fait à l'expert - en vertu de son seul savoir ou de sa seule technique dans une discipline), enfin au sens de vécu (Erlebnis).

14. À ce sujet, voir, outre l'article précédemment cité d'Agaesse et Sales (1932), ceux de Adnès (1932) et de Dupuy (1932), toujours dans le Dictionnaire de spiritualité ascétique et mystique. Par ailleurs, Bergamo (1994 [1991]) montre en quoi François de Sales a réussi à établir une nouvelle et désormais normative topique - psychologique plutôt qu'ontologique - de la mystique au XVII ${ }^{\mathrm{e}}$ siècle, après et contre celle, précédente, des rhéno-flamands. 
ment parlant; un idéalisme, pour parler en termes plutôt philosophiques, en est la condition.

Or, dans l'horizon du langage, l'intériorité peut et doit s'entendre d'une structure sujet-objet ou, si l'on préfère, du rapport même à l' «expérience ». Là, les termes en relation (sujet/objet, expérience/conscience/réel) prennent consistance et vérité à partir et en fonction de ce que réalise le geste originaire de la parole. C'est alors la nouveauté dans la manière d'exister d'un sujet vis-à-vis d'un objet et d'objets pour un sujet qui manifeste et marquent concrètement l'intériorité: elle qui procède d'une véritable altérité ${ }^{15}$. On comprend alors de quelle pseudo-altérité on se réclame quand celle-ci qualifie des «objets-en-soi », des « vécus», des «données de la conscience » ou une "expérience» traitée de façon surtout, voire exclusivement phénoménologique; cette altérité n'a plus qu'un sens métaphorique et donc une portée secondaire puisque l'autre — qu'il s'agisse d'objets ou bien de sujets - peut et doit être prédéterminé, en tenant précisément cet «état » pour le critère du savoir, de l'expérience, du réel, de la vérité... Au contraire, tout apparaît comme interprétation, fondamentalement, constitutivement, dans l'horizon du langage; à cet égard, une herméneutique de la spiritualité ne se contente pas de prendre acte de l'altérité mais y réside sans pouvoir y échapper. Dans ce désir qui brûle tout sauf luimême et qui brûle de lui-même, l'Autre peut très bien n'être qu'une réalité sans mots pour la dire ${ }^{16}$ puisqu'il demeure une expérience vécue dans et par un discours-qui-fait-expérience, c'est-à-dire en tant que geste de la

15. Maurice Zundel (1948 [1944]) a parlé peut-être plus poétiquement de ce régime d'altérité, de cette condition d'altérité: «La loi de cette assimilation [spirituelle] se formule aisément: que l'esprit garde vierge son intériorité, et que les objets revêtent eux-mêmes un mode intérieur qui lui donne prise sur eux. Ces deux conditions ne sont pas données, sinon à titre d'exigences. Leur réalisation constitue la dure conquête du savoir. La première est la plus difficile et la plus méconnue. Toutes les deux comportent des degrés qui font de la connaissance un progrès, et qui déterminent des différences de niveau, au point de vue de l'objet aussi bien qu'au point de vue du sujet. Ce sont ces différences de niveau [...] qui compliquent nos accords. On ne voit pas les mêmes choses à la même hauteur, ou l'on ne dirige pas, sur elles, un regard également réceptif: à moins que l'opposition, plus radicale encore, ne porte, à la fois, sur la signification des choses et sur la qualité du regard. » (p. 29) Il en a donné une formulation encore plus dense et concise: "Dans tout le champ de la vie spirituelle, l'altérité de l'objet n'est accessible qu'à l'altruisme du sujet. » (p. 42)

16. C'est indéniablement la conviction de Maurice Zundel, qui ne cesse de le rappeler et de l'exposer dans son œuvre. Le pas qu'il franchit par rapport à la théologie mystique, qui se limitait à poser un Dieu innommable (apophatisme), est donc bel et bien celui de soutenir qu'on n'a pas même besoin de nommer Dieu pour en vivre. Je ne 
parole. Le fin mot de la vie intérieure n'est donc pas un état de conscience mais un acte fondamental, (auto-)interprétatif et rigoureusement indéterminable: sinon comme mise en œuvre de l'Autre en soi. D'où le geste de la parole, qu'il importe d'exposer précisément à son titre de spiritualité depuis le début de mon propos. Ce déplacement herméneutique, pour saisir la spiritualité autrement qu'en termes strictement psychologiques, n'a pas consisté, si l'on en juge la démarche et les Actes du colloque en question, à congédier la phénoménologie mais à en suspendre le geste qui prétend aller par-delà le langage pour retrouver et isoler une "réalité idéale» ou une (a-)perception avant tout langage.

\section{La spiritualité comme pratique et compétence}

On l'a déjà compris: la parole dont il s'agit de rendre compte en son titre de spiritualité concerne un acte, plutôt qu'un contenu précis - sans rien n'exclure de ceci car c'est uniquement d'un renversement dont il est question. En parler comme d'une pratique de spiritualité permettrait de marquer «la spiritualité » à sa racine plutôt qu'en vertu d'une substantialisation, plus susceptible de nous éloigner du champ de la pratique qui a de tout temps servi à la situer. Cela permettrait en outre de la distinguer d'une pratique de "la spiritualité », qui signale une théorisation antécédente ou suréminente et qui appelle ensuite une mise en pratique. Et il s'agit certainement d'autre chose que de simples pratiques spirituelles, ces exercices ou activités désignés par leur ritualité et leur particularisme. Bref, la parole peut représenter une pratique de spiritualité à condition de sortir de l'opposition séculaire entre action et contemplation, de toute logique théoriepratique suivant une visée spéculative ou encore technique; inversement, une parole n'a plus grand-chose à voir avec une pratique de spiritualité quand elle se fait stratégie (technique) de communication pour livrer de simples informations, "passer des messages ", conduire autrui autant que le réel à ses seules vues et uniques intérêts.

Une façon de reconnaître la pratique de spiritualité que constitue la parole, c'est à ses fruits. Le principal et le premier de ces fruits, c'est l'instauration de sujets en acte et contemporains les uns des autres - plutôt qu'un savoir sur le monde et sur soi, sans en rien exclure. Un rapprochement entre personnes sur un plan affectif ou sur la base d'une "expé-

formule pas un enjeu différent en parlant, comme je le fais, de la spiritualité avant toute chose mais non sans la Chose. 
rience» semblable peut certes se produire; c'est tout de même dans leur être ensemble sujets de spiritualité, dans un savoir-devenir tels que se joue le geste de la parole entendu comme pratique de spiritualité. De fait, la démarche et les Actes du colloque ne véhiculent pas un faire-valoir relativement à des pratiques spirituelles particulières ou à ses expériences spirituelles personnelles; si on en parle, c'est de manière transitoire, en raison d'une pratique de spiritualité où chacun est mis en cause comme sujet dans et par la parole partagée. Là, l'intersubjectivité se manifeste au premier plan et comme la signature co-originelle d'une pratique. Quand le geste de la parole est élevé à cette pratique et vécu de la sorte, la posture des sujets est produite de l'intérieur même de leur pratique et indissociablement d'elle, par opposition à une position théorique ou pratique où le sujet pourrait encore se poser un tant soit peu de l'extérieur (en vertu d'une altérité métaphorique).

La pratique de spiritualité que peut constituer le geste de la parole s'éclaire aussi à partir de la compétence qu'elle requiert. Sans doute, le colloque en question suppose-t-il la compétence des personnes (laïques nommément), dont la prise de parole reste déterminante. Peut-être la metil justement de l'avant. Mais alors, la compétence dont il s'agit ne se réduit pas au savoir issu d'une formation particulière — sans l'exclure — pas plus qu'à une praxis s'imposant d'elle-même ou encore idéalement reflétée dans une théorie qui lui est conforme. À l'évidence, l'expert, que ce soit celui dans le domaine de la théologie spirituelle ou celui qui monnaye très bien son savoir-faire en vertu de techniques et donc d'une compétence professionnelle acquise, l'expert n'est pas la vedette en la matière. Les Actes du colloque reflètent d'ailleurs à quel point l'évocation des maîtres spirituels ou de "sa spiritualité à chacun" n'a pas servi à imposer une autorité ni à transmettre quelques vérités. Les échanges lors du colloque comme les discours spirituels visités (en l'occurrence) ont renvoyé à une compétence langagière, par tous partagée. Au travers du vécu et des attitudes, grâce à un savoir-être, la prise de parole s'est produite en une parole embrayée, autour de nos divers intérêts certes; se construit alors un monde (au sens heideggérien du terme), un monde ouvert et décentré (conformément à une Altérité radicale et véritable) par quoi se réfléchit une compétence (langagière) fondamentale.

La problématisation même de la spiritualité — dite laïque - dans les Actes du colloque qui nous intéressent donne une illustration concrète de cette pratique (de spiritualité) déployée en raison même de la compétence langagière. Des personnes laïques y parlent de spiritualité. Certes, des 
témoins les ont précédées à cet égard et ont même pu exceller en la matière: moines et moniales, religieux et religieuses, maîtres spirituels et mystiques. Pas question d'ignorer leur expérience ni de rejeter l'enseignement spirituel des Marie de l'Incarnation, Thérèse d'Avila ou Jean de la Croix. Mais la problématique du colloque ne concerne pas ces frontières comme telles et donc pas davantage une revendication par les laïcs d'une spiritualité qui leur serait spécifique ou spéciale. Le problème réside dans la surdétermination de la vie spirituelle, en l'occurrence en fonction de modèles de spiritualité qui correspondent pourtant à des situations particulières. Une idéalisation, voire une normalisation de la vie monastique/religieuse en serait la condition; l'essentiel, l'essence de la spiritualité aurait été donné et il ne resterait plus aux laïcs qu'à en faire des adaptations... notamment pour maintenir l'actualité de telle ou telle spiritualité. Cela seul garantirait une spiritualité vraie et orthodoxe de sorte que, pour les laïcs, il n'y aurait finalement des spiritualités que mimétiquement conçues et vécues ${ }^{17}$. Bref, on voit comment le problème a pu être posé d'un point de vue théorique (la doctrine dans telle spiritualité) puis pratique (applications, adapta-

17. Voir Cahiers (2005). Cette tendance a de quoi étonner quand on sait que les discours des maîtres spirituels modernes et donc l'expérience des spirituels de l'époque représentent précisément une sorte de réaction contre les institutions, ecclésiale autant que politique et académique; voir Michel de Certeau (1982) ou encore Jacques Le Brun (2002). Le contexte monastique, l'esprit de la communauté religieuse n'en est pas le ressort, au contraire de ce que l'on croit trop souvent.

Au risque de le répéter, la surdétermination de la spiritualité, qui prédomine actuellement, dépasse de loin cette assimilation à l'idéal monastique et se retrouve dans une vaste littérature non religieuse. Dans l'univers des soins de santé, la vie spirituelle est souvent décrite et conçue en parallèle ou même analogiquement à la vie biologique, à la vie morale, à la vie intellectuelle: suivant divers modèles explicatifs centrés sur un savoir permettant d'établir des données et de cerner des étapes de développement, pour l'efficacité d'interventions axées sur des compétences strictement professionnelles. On normalise toute la vie, y compris la vie spirituelle. Plus inquiétant encore, on en arrive parfois à faire dériver (les fonctions et repères de) la vie spirituelle (de ceux) de la vie intellectuelle ou morale, puis encore (de ceux) de la vie biologique. Tout fonctionne alors comme si les niveaux «inférieurs » de la vie représentaient une base explicative sûre et concrète pour comprendre les niveaux «supérieurs »; des fondations, des "données constitutives " sont déterminables de sorte qu'on peut expliquer puis gérer toutes les dimensions de la vie humaine (Pouliot, 2001). L'utilité, le succès de telles entreprises est indéniable; elles remplissent une fonction critique qui mérite de s'appliquer aussi à la spiritualité et à la religion. De là à dire que la spiritualité se trouve ainsi comprise en toute justesse et décrite avec une pertinence sans égale, rien n'est moins sûr. Il urge de rappeler le caractère toujours second de ces entreprises, par rapport au geste originaire de la parole interprétante. 
tions); on comprend en quoi a pu consister le refus dans la problématisation du colloque: une position de savoir déterminant les bonnes doctrines et le vrai vécu. Le défi consistait donc à pouvoir sortir ${ }^{18} \mathrm{du}$ sens ainsi donné à la situation pour déplacer la problématique dans une autre direction, avec une autre portée.

Tout compte fait, les Actes de ce colloque manifestent que des personnes laïques "parlent leur spiritualité ». Elles le font sans rançon à payer à l'égard de modèles-types, sans discours à emprunter pour reproduire l' "ordre spirituel » reçu. Là, elles n'inventent pas une autre spiritualité, dite laïque, voire séculière, après tant d'autres. Là, elles attestent autrement d'une pratique tout à fait humaine de spiritualité, grâce à une prise de parole et suivant cette modalité elle-même. Elles font concrètement «du spirituel ", elles montrent en quoi la spiritualité est un geste qui fait l'humain et son monde, quand bien même le rapport à l'absolu ne s'y trouve pas énoncé mais uniquement structuré (en une altérité fondamentale). Elles ravivent pratiquement du même coup qu'elles ramènent au regard cet enjeu (auto-)interprétatif qui nous constitue comme humain avec toute notre mondanéité (Heidegger, 1986 [1927]). Elles se retournent intérieurement, avec tout ce qu'elles produisent de contenus et de perspectives, de savoir et d'expérience, en leur geste de la parole; elles retournent au rapport autocritique et herméneutique en jeu dans leur «être ${ }^{19}$ », c'est-à-dire dans la mise en œuvre même du «spirituel ». Elles donnent donc à lire/écrire une existence en spiritualité, en raison d'une pratique de spiritualité exercée à l'intérieur du langage comme elle est liée de l'intérieur à la compétence langagière ${ }^{20}$. Et pareille pratique se fait manifestement discours autant qu'œuvre de signification.

18. Ce "pouvoir sortir» équivaut à une possibilité de l'ordre d'une compétence qui n'a rien de technique ou d'esthétique; cela s'associe à une performance consistant à signifier, à élaborer du sens autrement, conformément à l'enjeu dit de spiritualité qui est le cœur de mon propos.

19. «Être» au sens d'exister, le substantif étant relatif au verbe intransitif (exister) qui donne la consistance de l'être, de l'identité.

20. Maurice Zundel proposait la géniale formule d' «un au-delà au-dedans », qui a l'avantage - à mon avis — d'empêcher de poser une pure et totale extériorité sans sacrifier une pure et totale intériorité qui serait celle-là même de Dieu (voir par exemple Zundel 1967). Si mon propos s'inscrit dans le même sens, ma perspective est nettement poststructuraliste: ni psychologique ni idéaliste et encore moins esthétique. Je pousse plus loin, ou autrement, la recherche de l'intériorité, de l'intimité, d'un intérieur versus extérieur. Je ne cherche donc pas du tout à rejeter ou à m'affran- 


\section{Discours et signification}

On pourrait finalement résumer mon propos comme suit: le colloque en question sur la spiritualité dite laïque signale, par ses Actes, une mise en œuvre de la parole telle que celle-ci rend possible de ne pas en rester à la simple communication de connaissances sur "la spiritualité ", sans se résoudre à une esthétisation de la spiritualité ni au leurre d'une spécificité laïque en la matière. Pour comprendre la portée des discours qu'attestent ces Actes, il faut donc chercher plus loin que les énoncés qui les composent, je veux dire autrement qu'en vertu d'un tissu de données à déterminer puis à mettre en relation dans une discursivité systématique; pareil jeu de tissage est possible quand on tient les énoncés pour suffisants, pour normatifs par eux-mêmes, quand on les conçoit idéalement ou matériellement en eux-mêmes et pour eux-mêmes. L'idéalisme tout autant que le matérialisme ${ }^{21}$ empêcheront toujours de reconsidérer, de resituer, d'articuler pertinemment les énoncés dans l'énonciation qui les produit et le fait tenir ensemble. C'est pourquoi un discours n'est pas la somme de ses énoncés; une rhétorique pas plus qu'une esthétique du discours ne compenseront jamais cette conception, encore une fois parce que l'Altérité ne s'adonnerait aux discours que métaphoriquement - pas fondamentalement, pas comme radicalité herméneutique langagière. Un discours est certainement constitué d'énoncés et il est bien entendu opération de rationalités, mais si tout y est rationalité, la rationalité n'est pas tout. Le geste de la parole demeure, tel un au-delà à l'intérieur du discours et qui sourd de l'intérieur même du discours tout en l'excédant. D'où l' «effet» de subjectivité, l'«effet» d'objectivité, la «condition» de la pratique que j'ai auparavant exposé mais à titre d'exigences. Il est plus facile d'envisager d'abord des produits, des résultats, des énoncés ou des choses avant d'en (faire) voir les racines en termes de production, de construction, d'énonciation, de spiritualité mise en œuvre.

Un renversement semblable s'impose relativement au problème du sens, un renversement où se joue un véritable déplacement et que je mets en relief conformément au paradigme du langage. On peut bien parler de

chir de la tradition chrétienne à cet égard mais à montrer un déplacement — que la tradition mystique a appelé «extase» — jusque dans le paradigme du langage.

21. Idéalisme et matérialisme sont-ils autre chose que les deux facettes de la même médaille? Le matérialisme renverse l'idéalisme en le retournant parfois naïvement, parfois méthodiquement. Il faut encore remercier Feuerbach (1982 [1841]) pour sa démonstration à cet égard, quoiqu'il existe des argumentaires moins naifs, comme Zundel $(1960 ; 1967)$ en témoigne et en discute. 
sens (spécialement) spirituel ou de spiritualisation, tandis qu'on lit les Écritures ou qu'on parle de la vie humaine (laïque ou autre). Les Actes du colloque en ont tous les indices, mais «la dominante " n'est pas là, en un «sens" devant fonctionner uniquement à partir de données isolables et ensuite seulement à articuler. C'est plutôt l'articulation même du sens qui est en cause avec ce colloque, dans un horizon langagier. Rien à voir avec l'entreprise habituelle d'articuler du sens à la façon d'un "jeu de construction", où il suffirait d'organiser des «blocs" (idées, données, vécus, sentiments, etc.) dans des agencements toujours différents et selon des règles rhétoriques, esthétiques ou utilitaires - bref, en raison de règles exogènes au geste de la parole. Au lieu d'arrêter en bout de ligne le «sens " à quelque donnée à retrouver ou à décrypter, c'est le sens d'une donation qu'il s'agit constamment d'investir: en s'y retrouvant à la fois sous la modalité de sujet et celle d'objet ${ }^{22}$. Le geste de la parole permet de mieux comprendre que les façons de rendre compte de l'articulation du sens, à partir d'un sujet comme d'un objet, se partage entre celles, largement phénoménologiques, qui misent sur une méthode de corrélation et celles, dans l'orientation herméneutique qui est la mienne, qui révèlent et prennent acte de ce que toute corrélation est toujours déjà œuvre critique d'interprétation ${ }^{23}$.

Par articulation du sens, il faudrait alors entendre la construction même du sens, son advenir en nous et par nous, à travers tout ce qui s'élabore aussi en des gestes seconds et moins originaires tels que la connaissance scientifique ou autre. Le geste de la parole ne repose pas sur nos représentations du monde, de nous-mêmes, de Dieu, de la spiritualité, pas plus qu'il trouve là sa visée ultime et essentielle; en tout ceci se joue et se découvre plutôt l'inévitable mimesis (de: mimer!) qui sied à notre pensée et à notre action. L'acte de signifier ou, ce qui revient au même, une pratique signifiante mérite alors d'être mise en relief, par-devant ce caractère mimétique de notre penser et de notre agir qu'il s'agit de réinvestir sans déni. Ainsi peut-on élucider l'œuvre

22. Cette double modalité d'une donation a été décrite en termes de désir, voire de désir du désir, par Jacques Lacan (1975), en termes de croire par Michel de Certeau (1987), en termes de béance nihiliste par Nietzsche (1997 [1882]).

23. À ce point de mon propos, il conviendrait d'exposer en détail la théorie de la signification qui préside à l'approche sémiotique et qui sous-tend mes réflexions actuelles. Je me contente d'en annoncer les contours puisque le but de mon propos est seulement de signaler un déplacement dans la façon de se situer dans un comprendre procédant d'un interpréter. 
de la signification, cette semiosis qui (se) constitue (en) un acte en deçà de tout résultat du signifier en même temps qu'elle s'exhibe uniquement et précisément en la médiation - nécessaire et interne - du langage. Le geste de la parole en est à la fois l'instauration, la "re-présentation » et l'institution. Le "spirituel» relève toujours déjà d'une pratique signifiante; il tourne inévitablement en pratique signifiante; il est à la racine en même temps que le fruit de la réalité humaine - graciée par une Altérité qui, peut-être, ne sera jamais par nous nommée. De cette mimesis et de cette semiosis dépend la manière même dont on conçoit et exploite signes et symboles, mots et concepts, compréhension et interprétation - et dont on élucide le don (donné, donation). La langue française joue fort bien sur ce terme «signification ", qui correspond à un acte (de signifier) aussi bien qu'au résultat de cet acte (qu'on nomme signification); la question est de décider ce qui constitue la racine de toute "signification" et comment s'indique là une structure non phénoménologiquement prédéterminée.

\section{Résonances finales}

En désignant on ne peut plus clairement le geste de la parole, je tenais à sortir la parole de l'abstraction et de l'immatérialité où les apparences communes l'enferment de prime abord. J'ai voulu ainsi indiquer en quoi la parole mérite le titre de spiritualité et par quelle sortie ou en raison de quel déplacement cela devenait possible. C'est le sens de leur concrétude, de leur matérialité presque, qu'il me semble urgent de toucher: en les joignant de l'intérieur, l'une par l'autre. C'est-à-dire en en décidant dans le langage et donc sans autre extériorité qu'une altérité qui œuvre en fait uniquement du dedans, par le dedans.

À l'heure où de plus en plus de gens se réclament d'une spiritualité mais sans enracinement véritable dans quelque tradition religieuse tenue pour suspecte ou accessoire, tout autant que le corps d'ailleurs, on est saisi du défi de l'incarnation aussi bien que du risque de désincarnation pour nos contemporains. Notre parole, comme notre spiritualité, en est un symptôme.

À l'heure où l'on décrit couramment la crise du sens en s'inquiétant partout du manque ou de la perte de sens à la vie, comment ne pas être saisi par la marchandisation du sens, qui en multiplie la production au point que l'offre dépasse largement la demande: sans que les personnes se saisissent nécessairement et concrètement de/dans leur propre parole et 
spiritualité. Parole et spiritualité sont telle une vague; peut-on, ou non, vraiment y marcher ${ }^{24}$ ?

À l'époque qui est la nôtre, après le douloureux divorce entre théologie et spiritualité à l'aube de la modernité ${ }^{25}$ comme avec la séparation de la théologie et de la philosophie par la suite, on pourrait facilement avoir la nostalgie de la jadis unité - fusionnelle ? - de la théologie et de la spiritualité, de la théologie et de la philosophie. Toute tentative de restauration à cet égard me semble procéder de la conscience affolée devant l'ignorance, peut-être même dans le déni de la parole et de la spiritualité assumée en leur enjeu radicalement herméneutique parce que langagier. L'effort d'élucidation qui s'impose aurait avantage à s'opérer en l'accomplissement extatique — «désontologisé » et dépsychologisé — que les maîtres spirituels ont tout de même tracé pour nous.

\section{Références}

AdNÈs, P. (1932), «Mystique: II — Théories de la mystique chrétienne B. $\mathrm{XVI}^{\mathrm{e}}-\mathrm{Xx}^{\mathrm{e}}$ siècles ", dans M. VILlier, dir., Dictionnaire de spiritualité ascétique et mystique. Doctrine et histoire, Paris, Beauchesne, col. 19191939.

Agaesse, P. et M. Sales (1932), "Mystique: III — La vie mystique chrétienne », dans M. Villier, dir., Dictionnaire de spiritualité ascétique et mystique. Doctrine et histoire, Paris, Beauchesne, col. 1939-1983.

Bergamo, M. (1994 [1991]), L'anatomie de l'âme, Grenoble, Éditions Jérôme Million.

Cahiers de spiritualité ignatienne (2005): «Laïques et personnes consacrées: quel arrimage? ", 113.

— (2008): «Québec après 400 ans. Un terreau de spiritualité », 122.

- (2009a) : «Spiritualité sans religion», 124.

(2009b): «Notre spiritualité autrement... Prise de parole laïque sur la spiritualité chrétienne », 126.

Certeau, M. De (1982), La fable mystique: XVI $I^{e}-X V I I^{e}$ siècle, Paris, Gallimard.

24. Je fais évidemment allusion au récit évangélique de la marche sur les eaux par Pierre, là où Jésus, lui, marche sur la mer conformément à son attitude, à sa disposition signalée avec la prière en montagne: d'où il ne descend pas et en raison de quoi il ne coule jamais.

25. Voir Vandenbroucke (1950); Moioli (1987); Leclercq (1974). 
(1987), La faiblesse de croire, Paris, Seuil.

(2005), Le lieu de l'autre. Histoire religieuse et mystique, Paris, Gallimard/Seuil.

Delorme, J. (1992), "Sémiotique », dans J. Briend et E. Cothenet, dir., Dictionnaire de la Bible - Supplément, tome XII, p. 282-333.

Dumont, F. et al. (1971), Commission d'étude sur les lä̈cs et l'Église (Rapport Dumont). L'Église du Québec: un héritage, un projet, Montréal, Fides.

Dupuy, M. (1932), "Spiritualité: II. La notion de spirituel », dans M. Villier, dir., Dictionnaire de spiritualité ascétique et mystique. Doctrine et histoire, Paris, Beauchesne, col. 1160-1173.

Feuerbach, L. (1982 [1841]), L’Essence du christianisme, Paris, Éd. F. Maspero.

Heidegger, M. (1986 [1927]), Etre et temps, Paris, Gallimard.

HeISEnberg, W. (1972 [1932]), Les principes physiques de la théorie des quanta, Paris, Gauthier-Villars.

Kant, E. (1976 [1781]), Critique de la raison pure / trad. par J. Barni, Paris, Garnier- Flammarion.

Lacan, J. (1975), Le Séminaire. Livre XX: Encore, Paris, Seuil.

Le Brun, J. (2002), Le pur amour de Platon à Lacan, Paris, Seuil.

LECLERCQ, J. (1974), "Jalons pour une histoire de la théologie spirituelle», Seminarium, 26, p. 231-249.

Moioli, G. (1987), «Théologie spirituelle», dans S. de Fiores et T. Goffi, dir., Dictionnaire de la vie spirituelle, Paris, Cerf, p. 1120-1127.

Nietzsche, F. (1997 [1882]), Le gai savoir, Paris, GF Flammarion.

PAnier, L. (2008), «Figurativité - Discours — Énonciation », Sémiotique et Bible, 131, p. 29-45.

Pouliot, É. (2001), «Efficacité clinique des interventions médicales, infirmières, psychosociales et pastorales: mais à quelle condition? Bilan critique des recherches et publications sur le lien entre santé et spiritualité ", recherche et document préparés pour ce qui correspond aujourd'hui au Centre de pastorale de la santé et des services sociaux (CPSSS) de Québec.

SURIN, J.-J. (1963 [achevé en 1661, sans imprimatur]), Guide spirituel pour la perfection, Paris, Desclée de Brouwer. 
Vandenbroucke, F. (1950), "Le divorce entre théologie et mystique. Ses origines ", Nouvelle revue théologique, 72, p. 373-390.

Zundel, M. (1948 [1944]), L’homme passe l'homme, Paris, La Colombe. (1960), «Vrai et faux matérialismes », Choisir, 14, p. 15-20. (1962), Morale et mystique, Bruges, Desclée de Brouwer. (1967), L’homme existe-t-il?, Paris, Éditions ouvrières.

\section{Résumé}

L'adoption d'une perspective herméneutique plutôt que phénoménologique permet de décrire le «spirituel » en évitant toute substantialisation à cet égard, tant de «la spiritualité » elle-même que de ses soi-disant composantes. Une dépsychologisation devient ainsi possible, au bénéfice d'une présentation en termes de pratique de spiritualité (par opposition à pratiques spirituelles), de geste de la parole (en son titre même de spiritualité) et de pratique signifiante fondamentale.

\section{Abstract}

This paper shows how a hermeneutical paradigm is more appropriate than a phenomenological one for describing spirituality. Spirituality can then be understood without substantiating nor psychologising its presumed components. Practice of spirituality (vs spiritual practices), gesture (speech act, act of discourse) and fundamental signifying practice become key expressions to describe spirituality. 